\title{
Review: several non-pharmacological, pharmacological, and surgical treatments may be effective in urinary incontinence
}

Holroyd-Leduc JM, Straus SE. Management of urinary incontinence in women: scientific review. JAMA 2004;291:986-95.

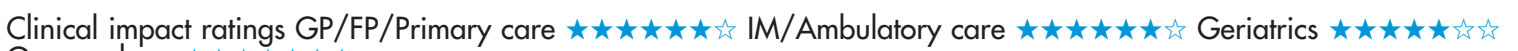

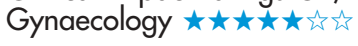

In women with urinary incontinence, which management strategies are effective?

\section{METHODS}

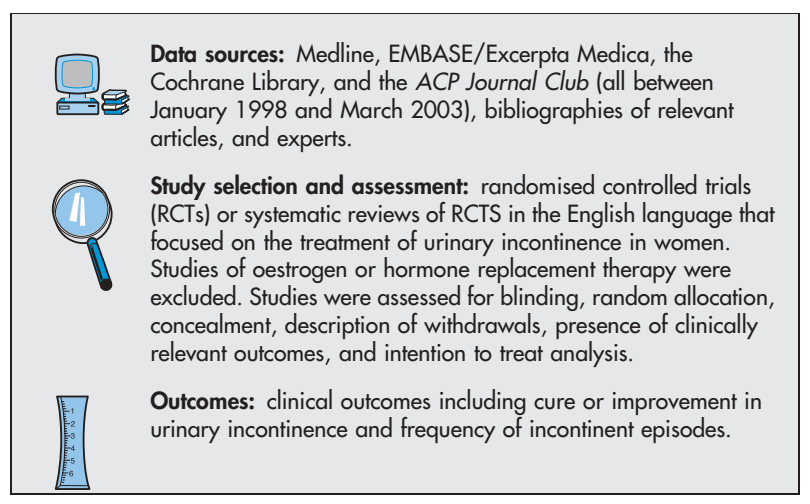

\section{MAIN RESULTS}

66 studies were included, of which 34 were treatment studies. A Cochrane review of trials in stress, urge, and mixed incontinence reported that pelvic floor muscle training (PFMT) increased self reported cure or improvement more than no treatment (relative risk [RR] 23.0, 95\% CI 7.6 to 70.2 ) or placebo (RR 1.5, CI 1.3 to 1.9). I RCT in women with stress or mixed incontinence reported that PFMT using a comprehensive clinic based programme was more effective at reducing the frequency of incontinent episodes than a self help booklet.

A Cochrane review reported that participants who received prompted voiding had fewer incontinent episodes in 24 hours (weighted mean difference [WMD] -0.93 , CI -1.32 to -0.53 ) than those in the control groups.

Combined Treatments. I RCT in women with stress, urge, or mixed incontinence reported greater self reported cure or improvement for PFMT combined with bladder training than for PFMT alone (RR 1.20, CI 1.02 to 1.42 ) or bladder training alone (RR 1.43, CI 1.17 to 1.74 ).

Devices. I RCT in patients with urge incontinence reported that electrical stimulation was more effective than sham stimulation for cure (no incontinence episodes and no detrusor overactivity on cystometry) (number needed to treat [NNT] 5, CI 3 to 42) and improvement (reduction in frequency of incontinence by $>50 \%$ or a cystometric bladder capacity increase $>50 \mathrm{ml}$ ) (NNT 2, CI 1 to 4). Vaginal cones appear to be inferior to PFMT and similar in effectiveness to electrical stimulation or other control interventions, but more data are needed. Data are limited but suggest that bladder training may be more effective than no bladder training for increasing subjective cure rates.

Medication. A Cochrane review reported that anticholinergic drugs for urge incontinence were more effective than placebo in subjective

For correspondence: Dr J M Holroyd-Leduc, Toronto General Hospital, Toronto, Ontario, Canada. jayna.holroyd-leduc@uhn.on.ca

Sources of funding: Veterans Affairs and Ontario Ministry of Health and Long-term Care. cure or improvement rates (RR 1.41, CI 1.29 to 1.54 ) and in improvement in leakage episodes in 24 hours (WMD -0.56 , CI -0.73 to -0.39), but a common adverse effect was dry mouth.

Surgery. Several surgical interventions including open retropubic colposuspension and the suburethral sling procedure may be effective for stress incontinence.

\section{CONCLUSIONS}

In women with urinary incontinence, non-pharmacological treatments including pelvic floor muscle training, electrical stimulation, bladder training, and prompted voiding may be effective. Anticholinergic drugs are effective for urge incontinence, and several surgical interventions (eg, open retropubic colposuspension and the suburethral sling procedure) may be effective for stress incontinence.

\section{Commentary}

U rinary incontinence has 2 main physiological mechanisms. In urge incontinence, the bladder muscle itself (detrusor) is unstable and starts to contract with small volumes of urine ( 0.5 cup) without activating the spinal reflex arc or having the reflex urge to void ignored. Specific anticholinergic drugs and anticholinergic side effects of other drugs are effective in increasing both the detrusor tone and urethral sphincter tone. In stress incontinence, the urethral sphincter has lost its muscle tone, and small bladder volume or pressures allow urine to leak. Surgical interventions altering the angle of the urethra to the bladder appear helpful.

The review by Holroyd-Leduc and Straus shows that evidence for primary prevention of urinary incontinence is for the woman never to deliver any infants; however, if pregnant, delivering by prophylactic caesarean sections for infants who are average to large weight appears helpful so that the pelvic muscles are not overly stretched for prolonged periods of time. Hysterectomies, medicines, comorbid diseases, age, and race are other factors evaluated independently in the literature and show association with incontinence development.

The non-pharmacological, non-surgical treatments reviewed in this article address general pelvic floor muscle weakness as 1 physiological deficit function in urinary incontinence. This review omitted some methods to improve incontinence that include physical activities such as yoga and Pilates, which can dramatically increase pelvic floor muscle tone, and local oestrogen treatment to increase sphincter tone. Methods that strengthen the pelvic floor muscles are effective if well taught and the patient is motivated to participate. However, often 1 or both components are missing. Primary care clinics may have limited referral capacity for these types of educational services; women may have little or no health insurance to pay for them; and well educated women may weigh the risks and benefits of pelvic floor muscle exercises compared with drug or surgery interventions and decide that the incremental gain from the exercise will not change their improvement with drug or surgical therapy. When possible, these methods should be developed and expertly taught. When not possible, drugs and surgery are the mainstay of treatment options.

Diane M Harper, MD, MPH, MS Dartmouth Medical School Hanover, New Hampshire, USA 